تأثير التغذية المثقطعة في كفاءة اداء وحدات الحمأة المنشطة ذات المزج القطاعي العاملة بأوقات مكوث هيدروليكية متغيزة

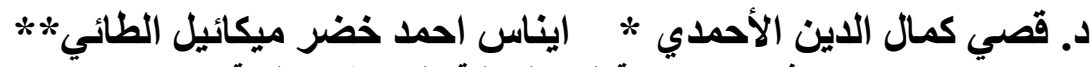

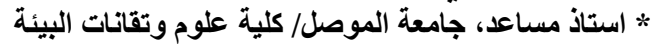

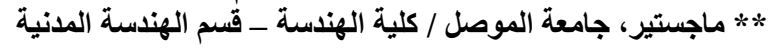

\title{
الخلاصة
}

تم في هذا البحث دراسة تأثير التغذية المتقطعة في كفاعة واستقرارية أنظمة الحمأة المنشطة ذات الجئة الجريان

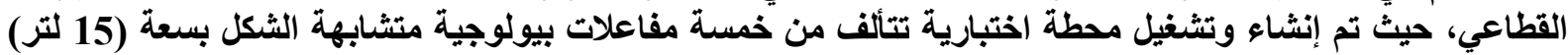

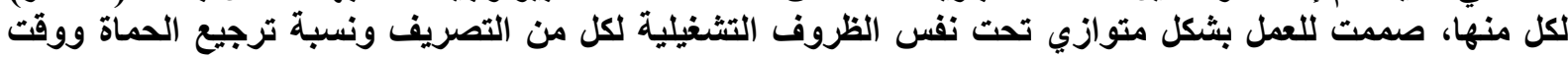

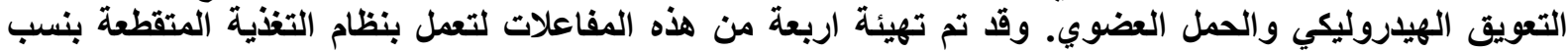

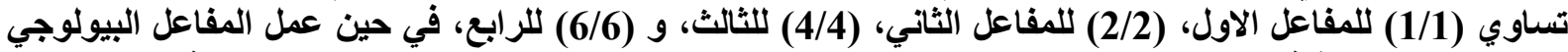

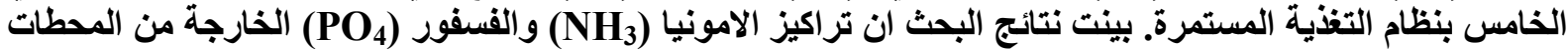

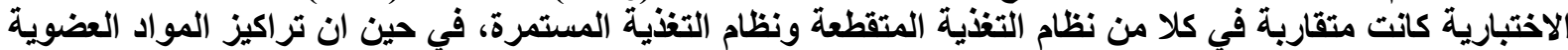

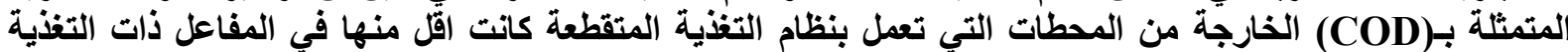

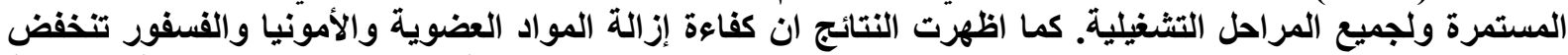

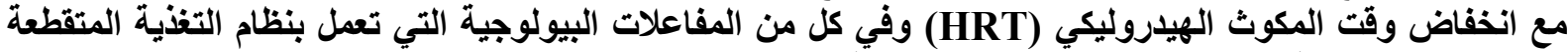

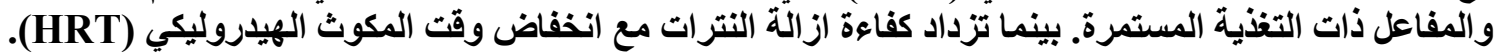

Keywords: Plug flow, Activated sludge, intermittent feeding, Intermittent aeration, Hydraulic retention time.

\section{Effect of Intermittent Feeding on the Performance of Plug Flow Activated Sludge System under Different Hydraulic Retention Times}

\author{
Dr. Kossay K. Al-Ahmady* Enas Ahmad Kader AL-Taee** \\ * Asst. Professor, College of Environmental Science and Technology, University of Mosul \\ ** M.Sc., Civil Dept. College of Engineering, University of Mosul
}

\begin{abstract}
In this research, the effect of intermittent feeding on the efficiency of plug flow activated sludge reactors has been studied. An experimental plant has been constructed and operated. The plant consists of five identical reactors with the volume of (15) liter working in parallel under the same operating conditions for each of discharge; sludge recycling ratio; hydraulic detention time and the organic load. Four of these reactors had been worked at the intermittent (ON/OFF) feeding mode with the ratios of (1/1), $(2 / 2),(4 / 4)$ and (6/6) for the first, second, third, and fourth reactor respectively, while the fifth reactor worked at continuous feeding mode. The results showed that, removal efficiencies for each of ammonia $\left(\mathrm{NH}_{3}\right)$ and phosphorus $\left(\mathrm{PO}_{4}\right)$ were close in both of intermittent and continuous feeding system, while the concentrations of (COD) in effluent were less in the intermittent reactors. Removal efficiencies of (COD), $\left(\mathrm{NH}_{3}\right)$, and $\left(\mathrm{PO}_{4}\right)$ decreased with reducing the hydraulic retention, whereas nitrate removal efficiency increased with decreasing of hydraulic retention time.
\end{abstract}


يعتبر نظام الحمأة المنشطة أحد أنظمة النمو العالق، وقد تم اشتقاق اسم الحمأة المنشطة في هذا النوع من الأنظمة

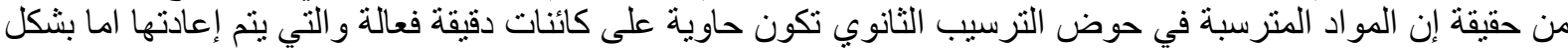

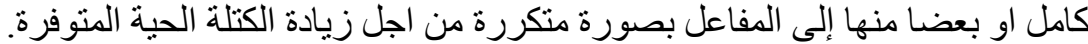

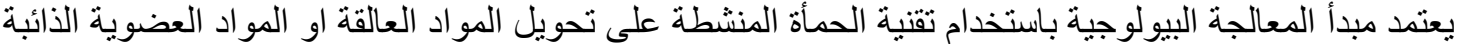

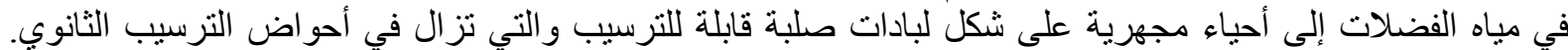
وكحال بقية أنظمة المعالجة البيولوجية فقد تطورت أنظمة الحمأة المنشطة من أسلوب التشغيل باءلئ بالدفعات (Batch reactor)

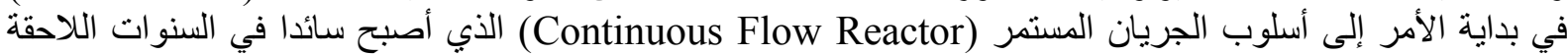

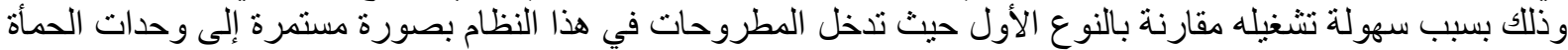

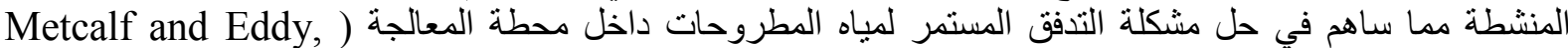

ومع الوقت ظهرت العديد من التعديلات على نظام الحمأة المنشطة مثل نظام قنوات الأكسدة ونظام التهوية

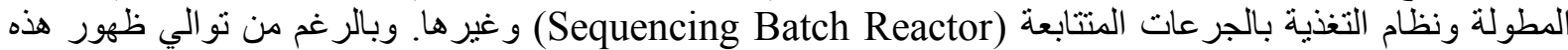

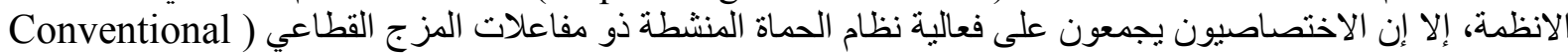

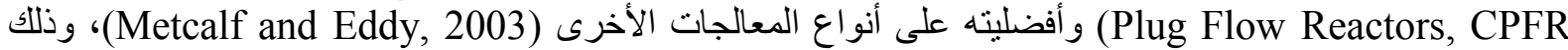

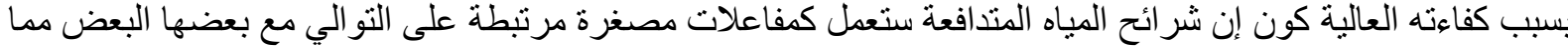

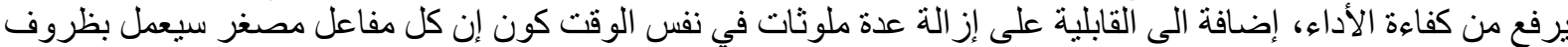

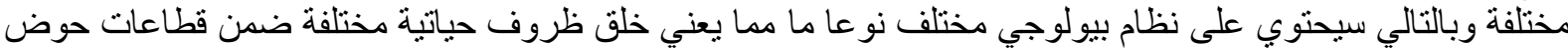

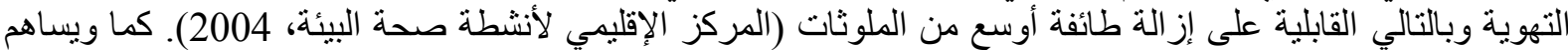

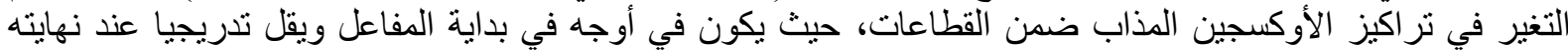

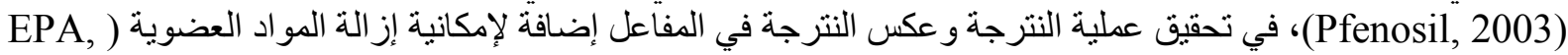

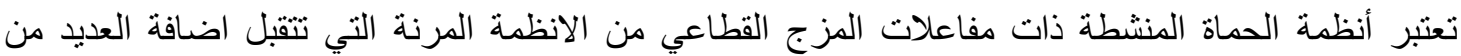

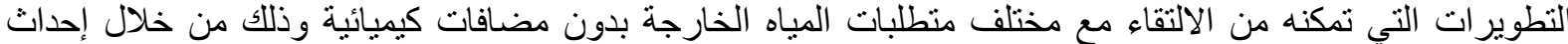

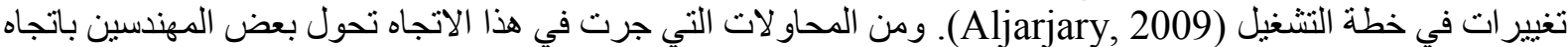

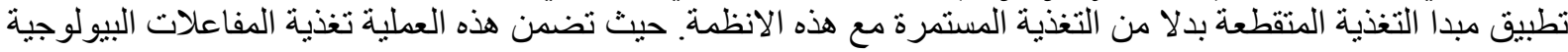

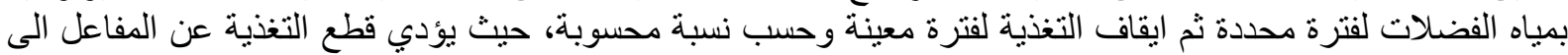

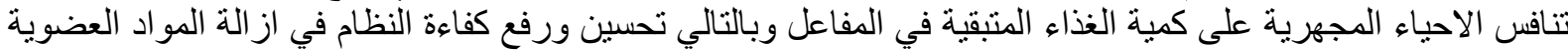

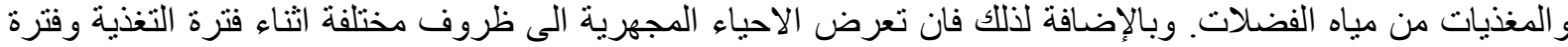
انقطاعها وبشكل دوري يساعد في منع حصول ظاهرة انتفاخ الحمأة في هذا النظام وبالتالي الحصول على التى حمأة ذات

خصائص ترسيبيه جيدة (Metcalf and Eddy, 2003). يهدف هذا البحث الى دراسة تأثير طول مدد تشغيل التغذية ومدد انقطاعها في كفاءة إزالة الموات التواد العضوية

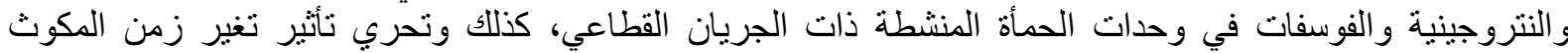

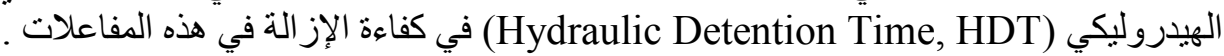
الار اسات السابقة

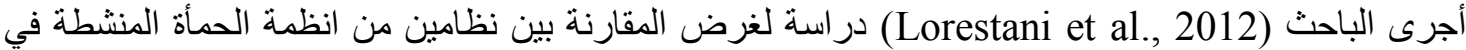

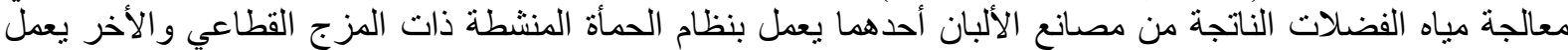

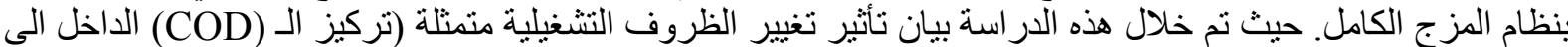

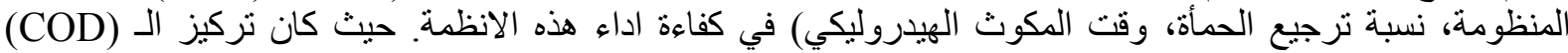

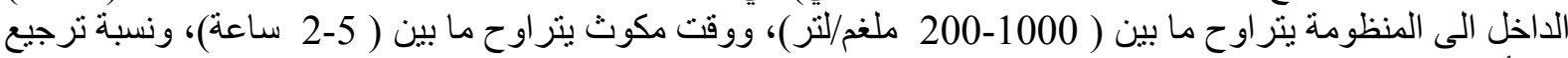

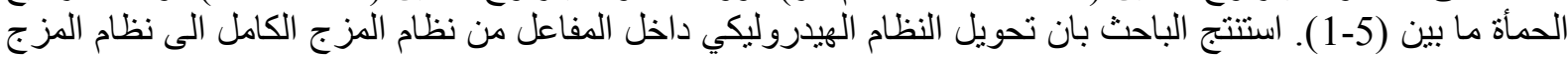

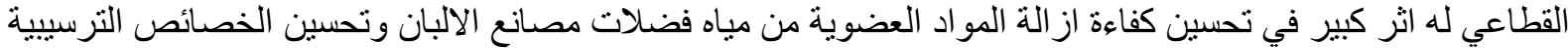

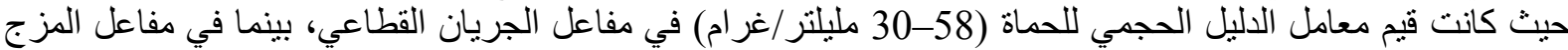
الكامل تتر اوح بين (145-50 مليلتر /غر ام). وقد قام الباحث (Chelliapan et al., 2011) بدراسة تأثير تغيير الظروف التشات التشغيلية (الحمل الهيدروليكي

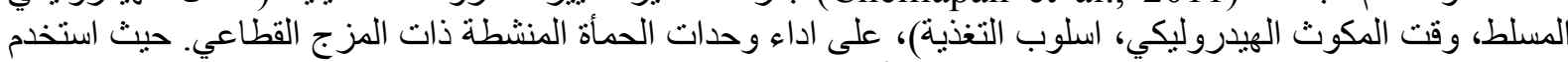

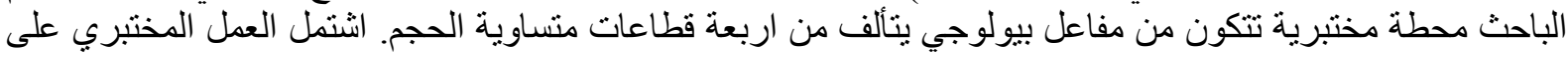




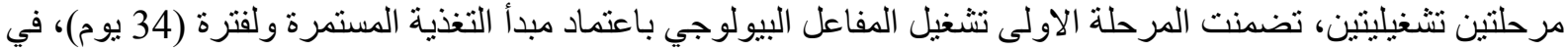

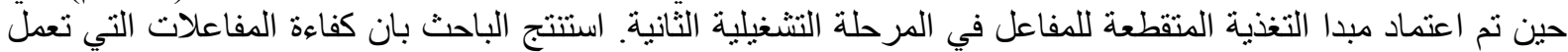

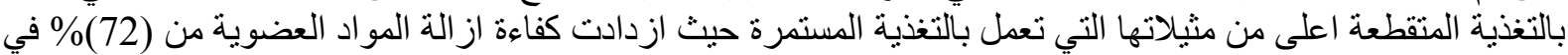

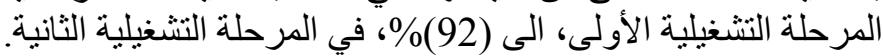

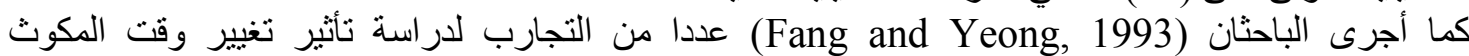

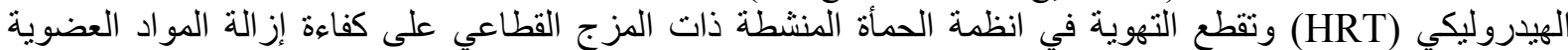

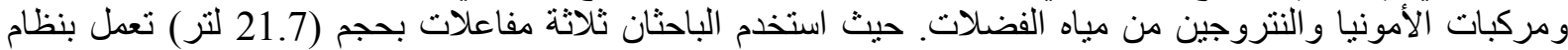

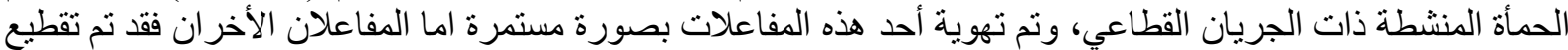

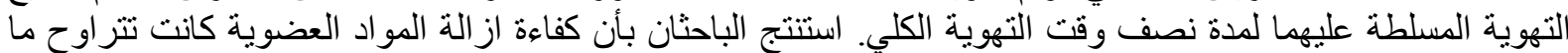
بين (197\%-95) وكفاءة از الة الامونيا تساوي (85\%).

\section{المواد وطرائق العمل المبّري}

انشاء المحطة الاختبارية (المفاعلات البيولوجية الاختبارية)

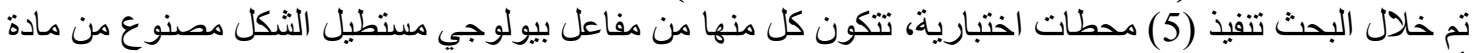

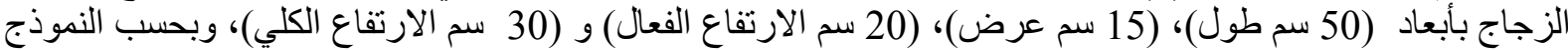

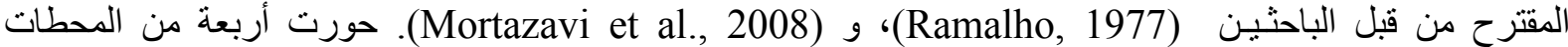
الاختبارية لتعمل بنظام الحمأة المنشطة ذات الجريان القطاعي (Plug flow) والتخذية المتقطعة ( Intermittent)

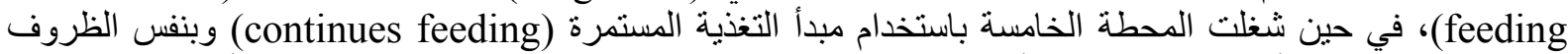

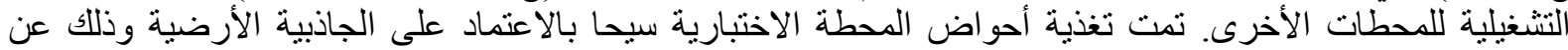

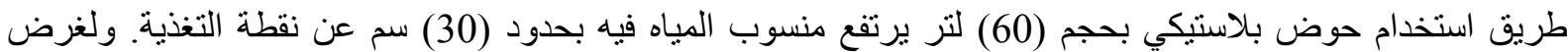

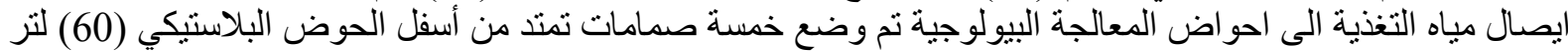

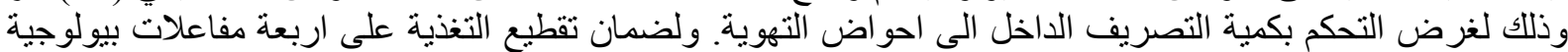

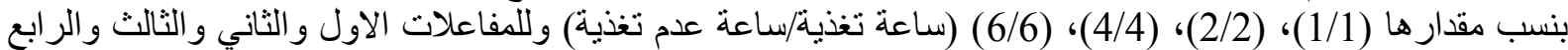

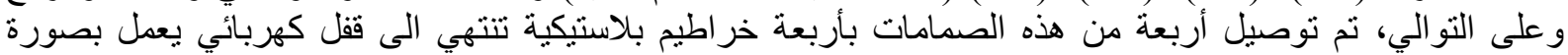

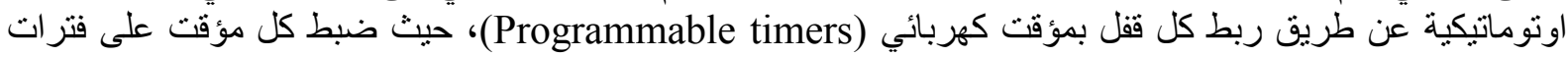
تشغيل و انطفاء محددة حسب نسية نسبة التغذية الى انقطاعها في كل مفاعل من المفاعلات الاربعة، اما المفاعل الخامس فيتم تغذيته من الحوض البلاستيكي بصورة مستمرة.

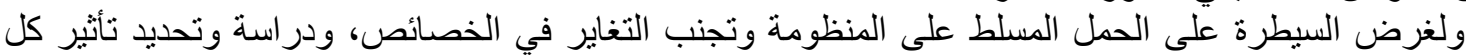
متغير بشكل مستقل بدون التداخل الحاصل عن تغير نوع وخصائص مياه التهاه الفضلات الداخلة فقد استخدم في البحث مياه

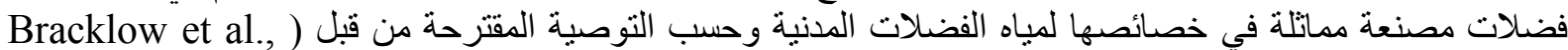
2007). يبين الجدول رقم (1) المو اد المستخدمة في صناعة مياه الفضلات الثبيهة بمياه الفضلات المنزلية فئل

الجدول رقم (1): المواد المستخدمة في صناعة مياه الفضلات الثبيهة بمياه الفضلات المنزلية .(Bracklow et al., 2007)

\begin{tabular}{|c|c|c|c|c|c|}
\hline الفسفور الكلي & النتروجين الكلي & $\begin{array}{l}\text { BOD5 } \\
(\mathrm{mg} / \mathrm{l})\end{array}$ & $\begin{array}{l}\text { COD } \\
(\mathrm{mg} / \mathrm{l})\end{array}$ & $\begin{array}{l}\text { التركيز } \\
\text { (mg/l) }\end{array}$ & المواد المستخدمة \\
\hline 0 & 27 & 0 & 0 & 104 & $\mathrm{NH}_{4} \mathrm{CL}$ \\
\hline 0 & 1 & 14.5 & 25.9 & 25.9 & Peptone \\
\hline 4.3 & 0 & 0 & 0 & 19.35 & $\mathrm{KH}_{2} \mathrm{PO}_{4}$ \\
\hline 0 & 0 & 153.6 & 271 & 271 & النشأ \\
\hline 1.96 & 11.96 & 116 & 200 & 200 & Milk \\
\hline 1.74 & 0 & 0 & 0 & 10.034 & $\mathrm{~K}_{2} \mathrm{HPO}_{4}$ \\
\hline 0 & 0 & 0 & 0 & 5.8 & $\mathrm{MgSO} 4.7 \mathrm{H}_{2} \mathrm{O}$ \\
\hline 0 & 21.4 & 6.83 & 11.61 & 45.87 & Urea \\
\hline 8 & 40 & 300 & 500 & & المجموع \\
\hline
\end{tabular}


تم تشغيل المحطات الاختبارية الخمسة على ثلاثة مر احل تشغيلية. تمت تغذية احدى هذه المحطات بشكل مستمر

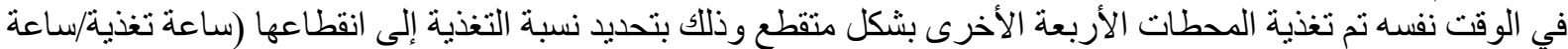

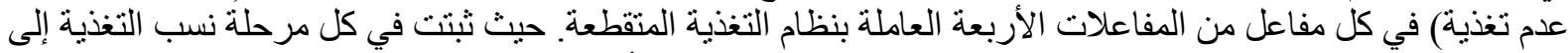

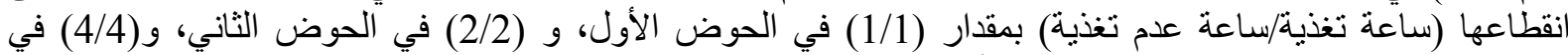

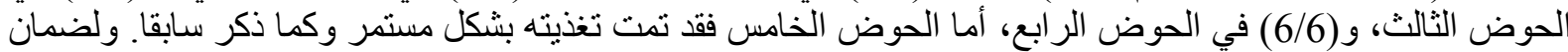

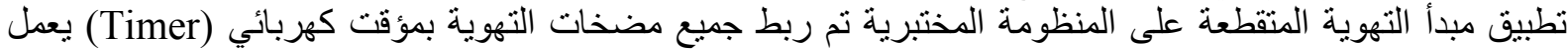

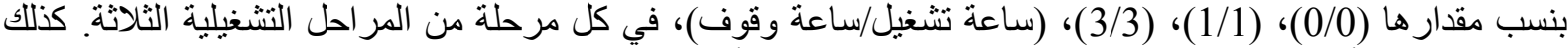
ولغرض تحقيق أهداف البحث فقد شغلت المحطات الخمس على أزمان مكوث هيدروليكية مختلفة تتز اوح ما بين (12) إلى الى

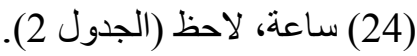
تمت مراقبة كل من نراكيز المنطلب الكيميائي للأوكسجين الذائب (COD

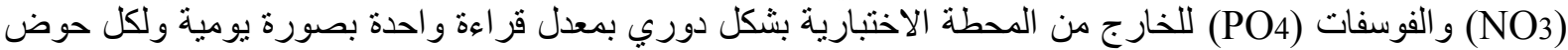
وذللك لتقييم كفاءة الإز الة (Ramalho,1977).

الجدول رقم (2): يبين المراحل التشغيلية للمحطة الاختبارية

\begin{tabular}{|c|c|c|c|c|c|c|c|c|}
\hline تثنغيل/توقف الخامس & 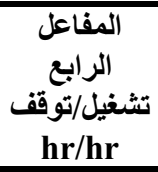 & تثغيل/توقلف الثالث & تثغيل/توقلف الثناني & $\begin{array}{c}\text { تثغيل/توقفل الاول } \\
\text { hr/hr }\end{array}$ & تثغيل/وقوفية & 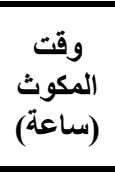 & الجولة & التشغيلية \\
\hline متقطع & متقطع & متقطع & متقطع & مستمر & مستمرة & \multirow{6}{*}{12} & الاولى & \multirow{6}{*}{ الاولى } \\
\hline $6 / 6$ & $4 / 4$ & $2 / 2$ & $1 / 1$ & $0 / 0$ & $0 / 0$ & & & \\
\hline متقطع & متقطع & متقطع & متقطع & مستمر & متقطعة & & الثانية & \\
\hline $6 / 6$ & $4 / 4$ & $2 / 2$ & $1 / 1$ & $0 / 0$ & $1 / 1$ & & & \\
\hline متقطع & متقطع & متقطع & متقطع & مستمر & متقطعة & & الثالثة & \\
\hline $6 / 6$ & $4 / 4$ & $2 / 2$ & $1 / 1$ & $0 / 0$ & $3 / 3$ & & & \\
\hline متقطع & متقطع & متقطع & متقطع & مستمر & مستمرة & \multirow{6}{*}{18} & الر ابعة & \multirow{6}{*}{ الثانية } \\
\hline $6 / 6$ & $4 / 4$ & $2 / 2$ & $1 / 1$ & $0 / 0$ & $0 / 0$ & & & \\
\hline متقطع & متقطع & متقطع & متقطع & مستمر & متقطعة & & الخامسة & \\
\hline $6 / 6$ & $4 / 4$ & $2 / 2$ & $1 / 1$ & $0 / 0$ & $1 / 1$ & & & \\
\hline متقطع & متقطع & متقطع & متقطع & مستمر & متقطعة & & السادسة & \\
\hline $6 / 6$ & $4 / 4$ & $2 / 2$ & $1 / 1$ & $0 / 0$ & $3 / 3$ & & & \\
\hline متقطع & متقطع & متقطع & متقطع & مستمر & مستمرة & \multirow{6}{*}{24} & السابعة & \multirow{6}{*}{ الثالثة } \\
\hline $6 / 6$ & $4 / 4$ & $2 / 2$ & $1 / 1$ & $0 / 0$ & $0 / 0$ & & & \\
\hline متقطع & متقطع & متقطع & متقطع & مستمر & متقطعة & & الثامنة & \\
\hline $6 / 6$ & $4 / 4$ & $2 / 2$ & $1 / 1$ & $0 / 0$ & $1 / 1$ & & & \\
\hline متقطع & متقطع & متقطع & متقطع & مستمر & متقطعة & & التاسعة & \\
\hline $6 / 6$ & $4 / 4$ & $2 / 2$ & $1 / 1$ & $0 / 0$ & $3 / 3$ & & & \\
\hline
\end{tabular}

التتائج والمناقشة

تأثير تغير وقت المكوث الهيدروليكي في كفاعة ازالة المواد العضوية العاية وكفاعة ازالة الامونيا

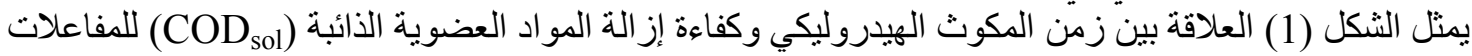

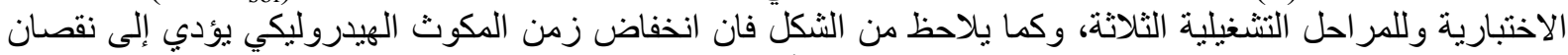

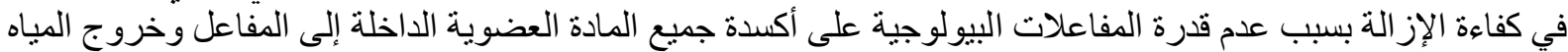

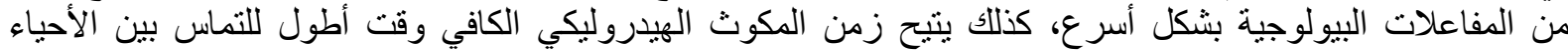

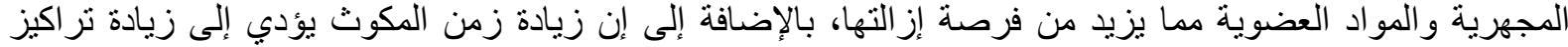

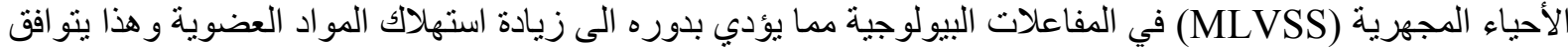

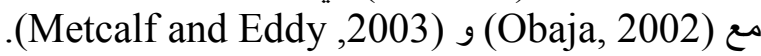

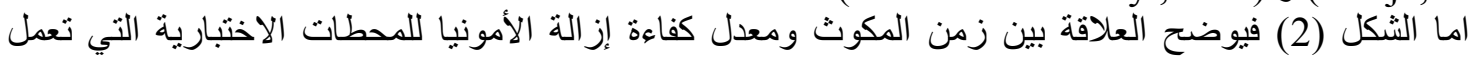

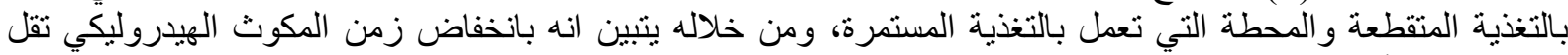

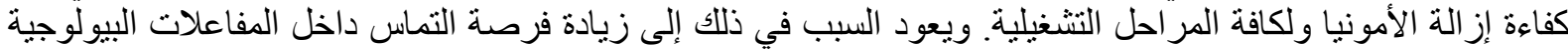


مع زيادة زمن المكوث الهيدروليكي مما يزيد من فرصة قيام الأحياء المجهرية بأكسدة الأمونيا إلى النتريت بعملية النترجة، وتتو افق هذه النتيجة مع (Obaja, 2002)

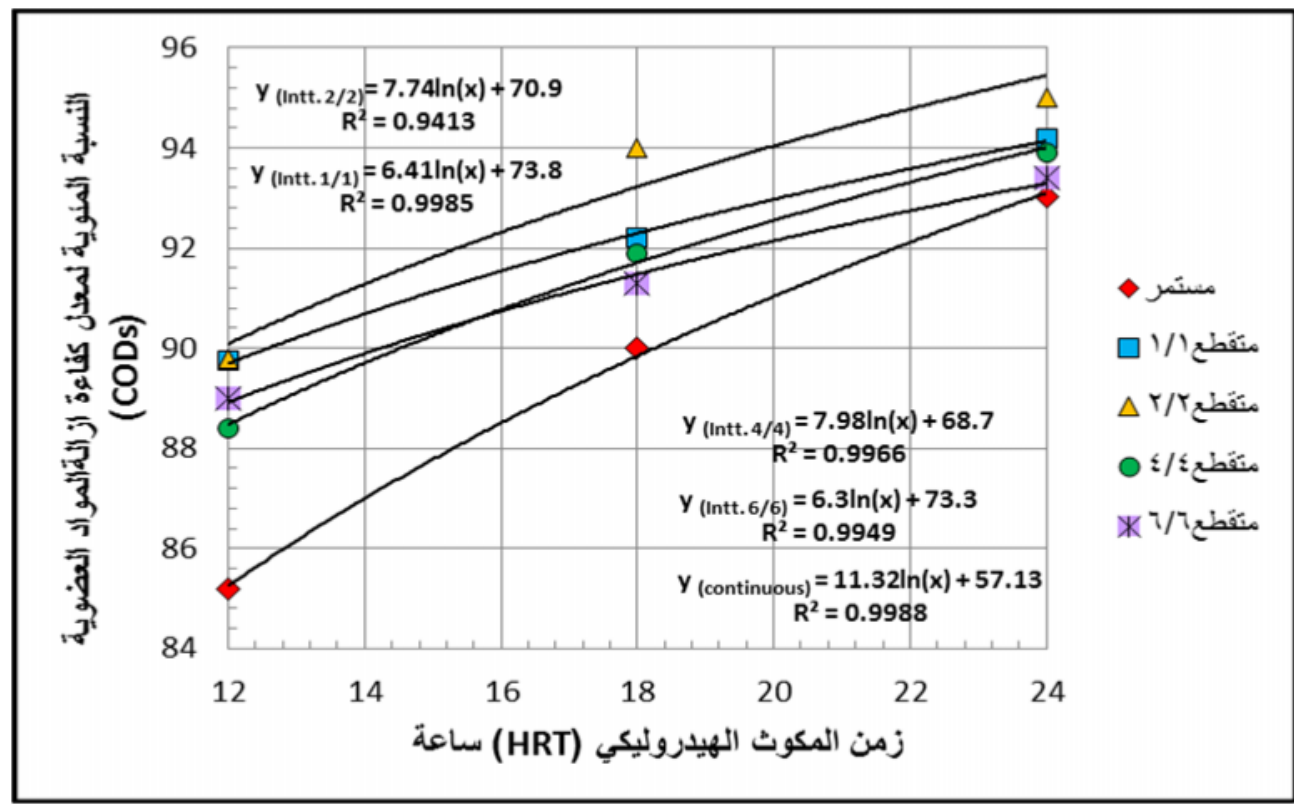

الثكل (1): العلاقة بين زمن المكوث الهيدروليكي (HRT) وكفاعة إزالة المواد العضوية الذائبة (COD sol) الاختبارية وللمر احل التثغيلية الثلاثة.

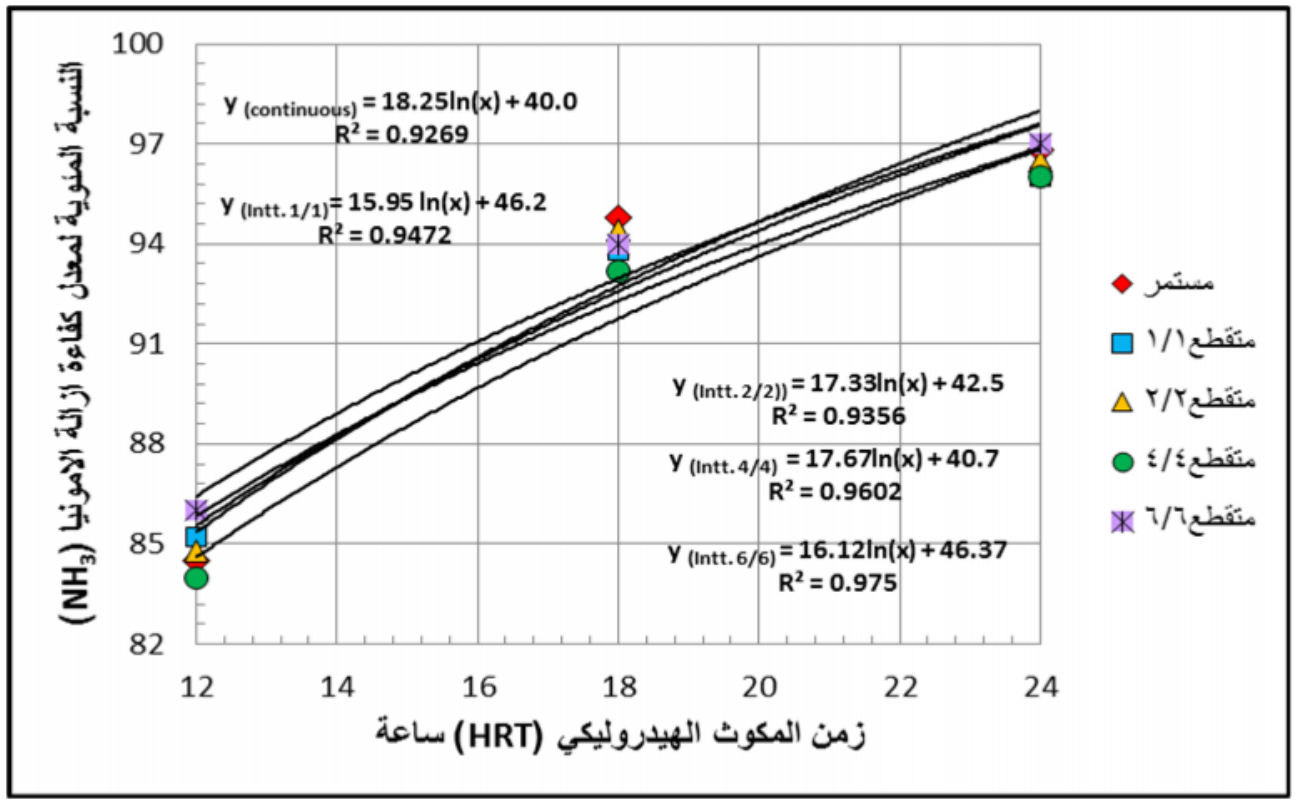

الثكل (2): العلاقة بين زمن المكوث الهيدروليكي ومعدل كفاءة إزالة الأمونيا (NH3).

تأثير تغير زمن المكوث الهيلروليكي في تراكيز الفوسفات (PO4)

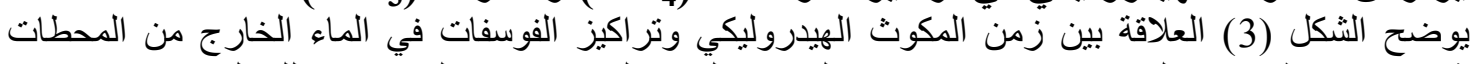

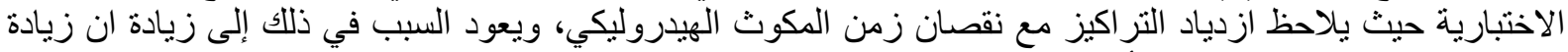

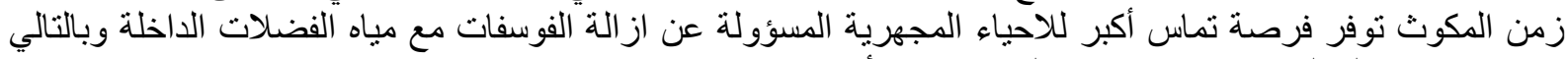

زيادة فرصة إز الة الفوسفات، وتتفق هذه النتيجة مع ما أورده (Garcia et al., 2002). 
اما بالنسبة لتأثير زمن المكوث الهيدروليكي في تر اكيز النترات الخارجة من المحطات الاختبارية التي تعمل بنظام

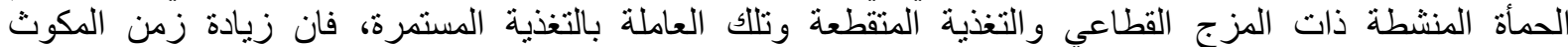

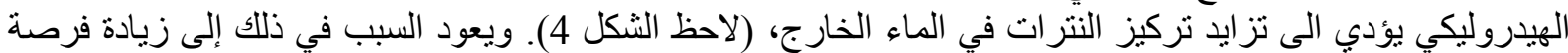

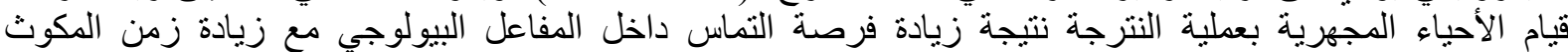
الهيدروليكي، وتتو افق هذه النتيجة مع ما أورده العديد من الباحثين مثل (Fang and Yeong, 1993).

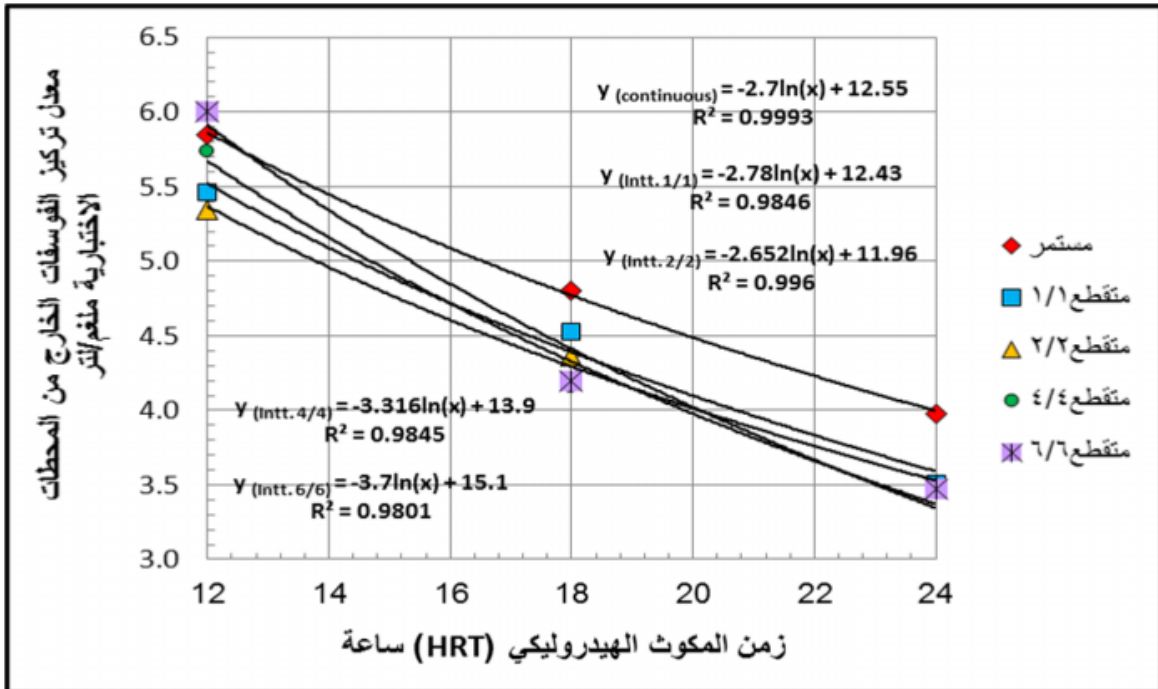

الثكل (3): العلاقة بين زمن المكوث الهيدروليكي (HRT) للمفاعل البيولوجي ومعدل تركيز الفوسفات المتبقي الخارج من المحطات الاختبارية.

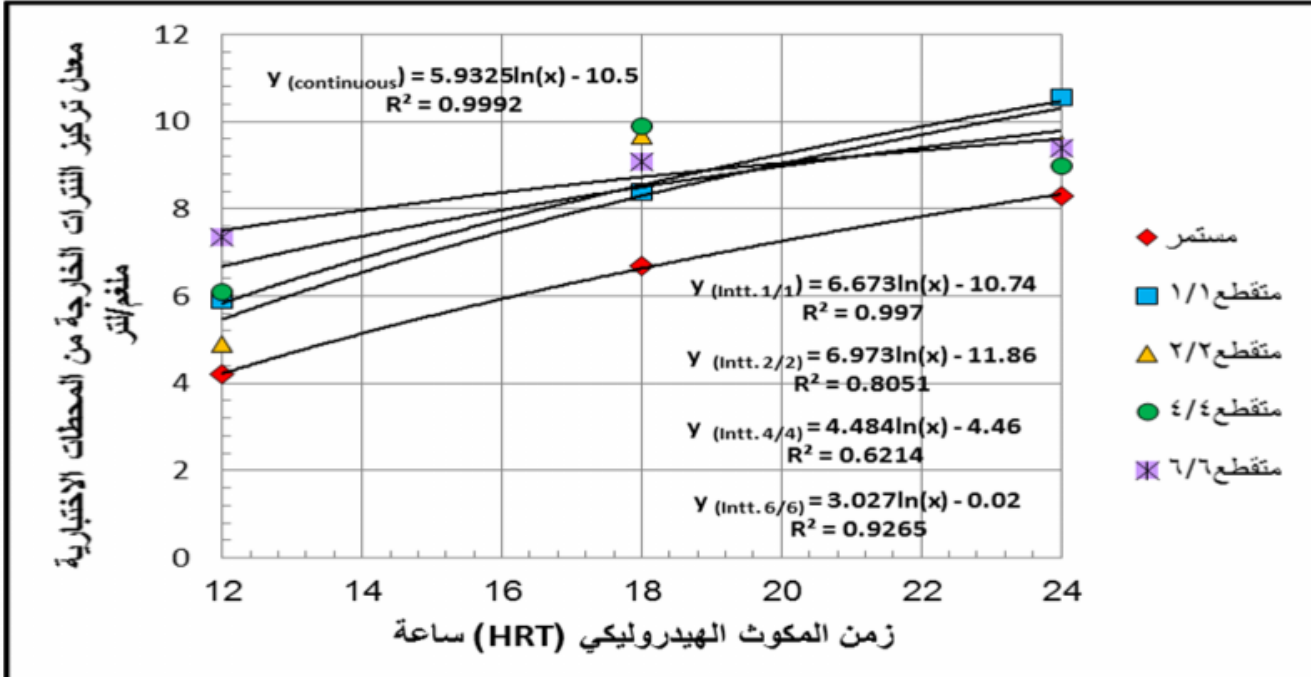

الثكل (4): العلاقة بين زمن المكوث الهيدروليكي (HRT) ومعدل تركيز النترات الخارجة من المحطات الاختبارية.

تأثير تغير اسلوب التغذية في كفاءة ازالة المواد العضوية

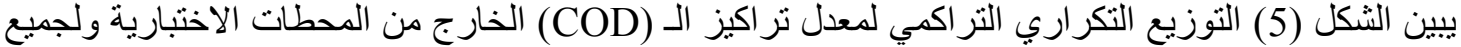

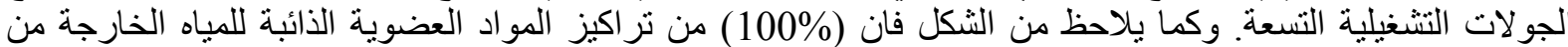
المحطات ذات التغذية المتقطعة والمحطة ذات التغذية المستمرة على التو الي كانت ضدن الترن محددات الطرح المحلية (وزارة

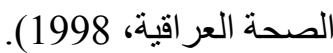




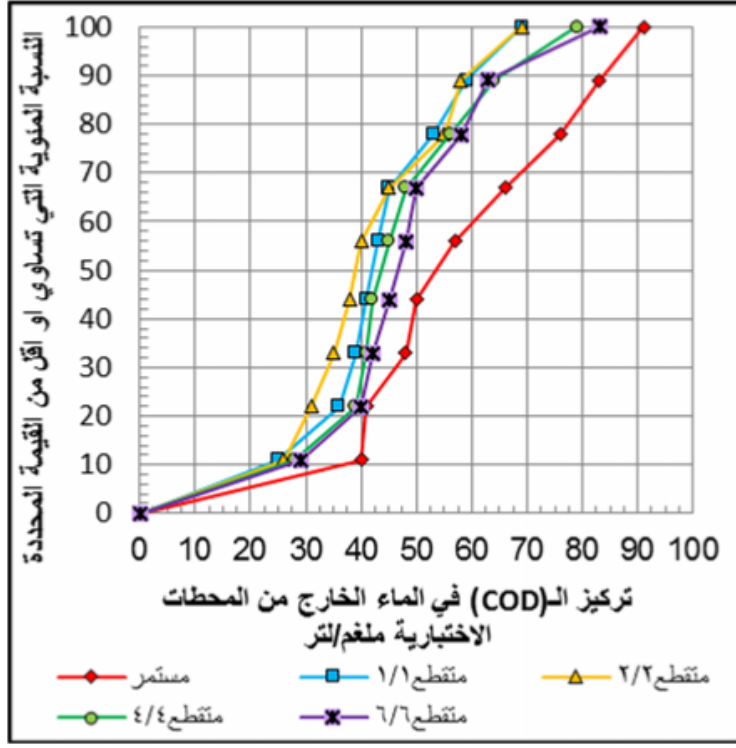

التَكل (5): التوزيع التكراري التراكمي لمعل تراكيز المواد العضويه الداتبه الخارجه وللمراحل التتنغيليه التلات

تأثثير تغير اسلوب التغذية في كفاعة ازالة المواد التيثروجينية

يظهر الثكل (6) ان (22\%) من نر اكيز الأمونيا الخارجة من مفاعلات الجريان القطاعي ذات التغذية المتقطعة

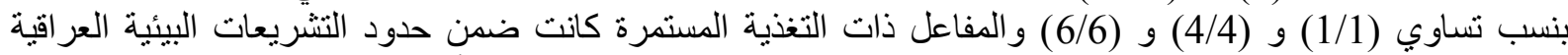

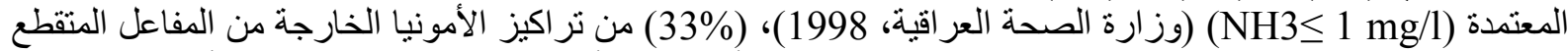

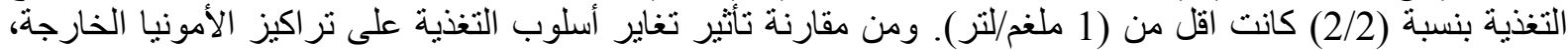

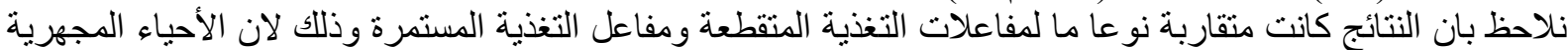

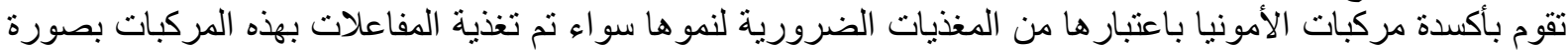

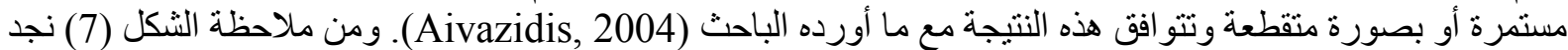

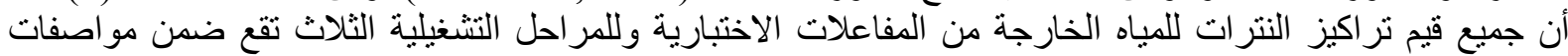

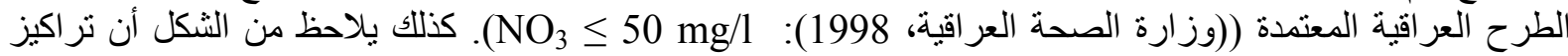

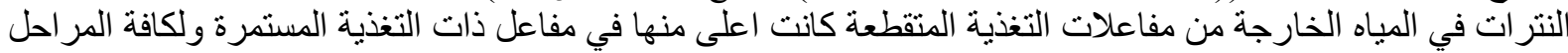

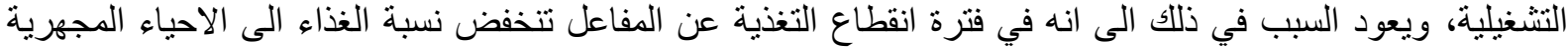

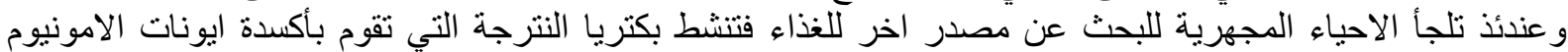
إلى نتريت $\left(\mathrm{NH}_{4}{ }^{+}\right)$

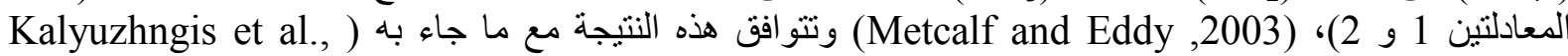

$$
\begin{aligned}
& 55 \mathrm{NH}_{4}{ }^{+}+76 \mathrm{O}_{2}+109 \mathrm{HCO}_{3} \stackrel{- \text { Nitrosomonas }}{\longrightarrow} \mathrm{C}_{5} \mathrm{H}_{7} \mathrm{NO}_{2}+54 \mathrm{NO}_{2}{ }^{-}+57 \mathrm{H}_{2} \mathrm{O}+104 \mathrm{H}_{2} \mathrm{CO}_{3} \ldots . .(1) \\
& 400 \mathrm{NO}_{2}{ }^{-}+\mathrm{NH}_{4}{ }^{+}+4 \mathrm{H}_{2} \mathrm{CO}_{3}+\mathrm{HCO}_{3}{ }^{-}+195 \mathrm{O}_{2} \stackrel{\text { Nitrobactor }}{\longrightarrow} \mathrm{C}_{5} \mathrm{H}_{7} \mathrm{NO}_{2}+3 \mathrm{H}_{2} \mathrm{O}+400 \mathrm{NO}_{3}{ }^{-} \ldots . .(2)
\end{aligned}
$$

تأثير تغير نظام التهوية في تراكيز الفوسفات والنترات الخارجة من المحطات الاختبارية

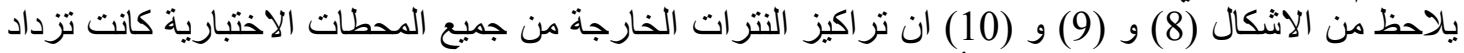

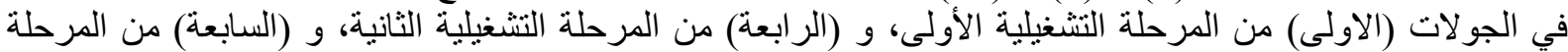

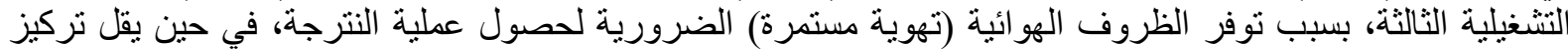

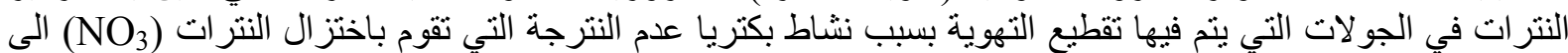

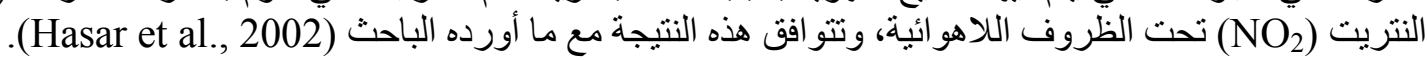


كذللك تبين الاشكال (11) و (12)وو(13) ان نركيز الفوسفات الخارج من جميع المحطات الاختبارية كان

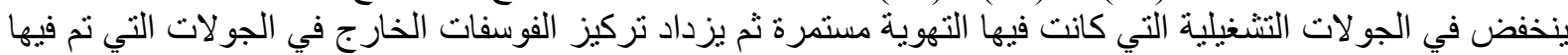

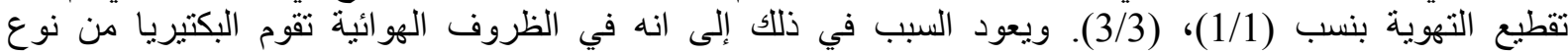
(Heterotrophic)

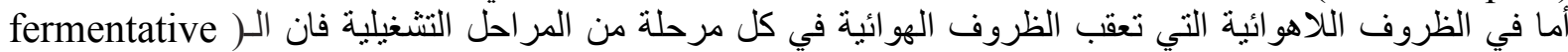
(bacteria

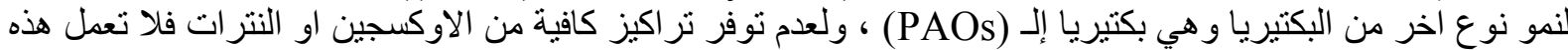

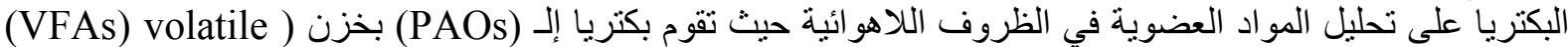
(fatty acids وتحرير الفسفور الذائب والمخزون في الخلية البكتيرية، وتنتوافق هذه النتيجة مع ما أورده الباحثون

(Garcia et al., 2003) ،(Ekama and Wentzel, 1999)
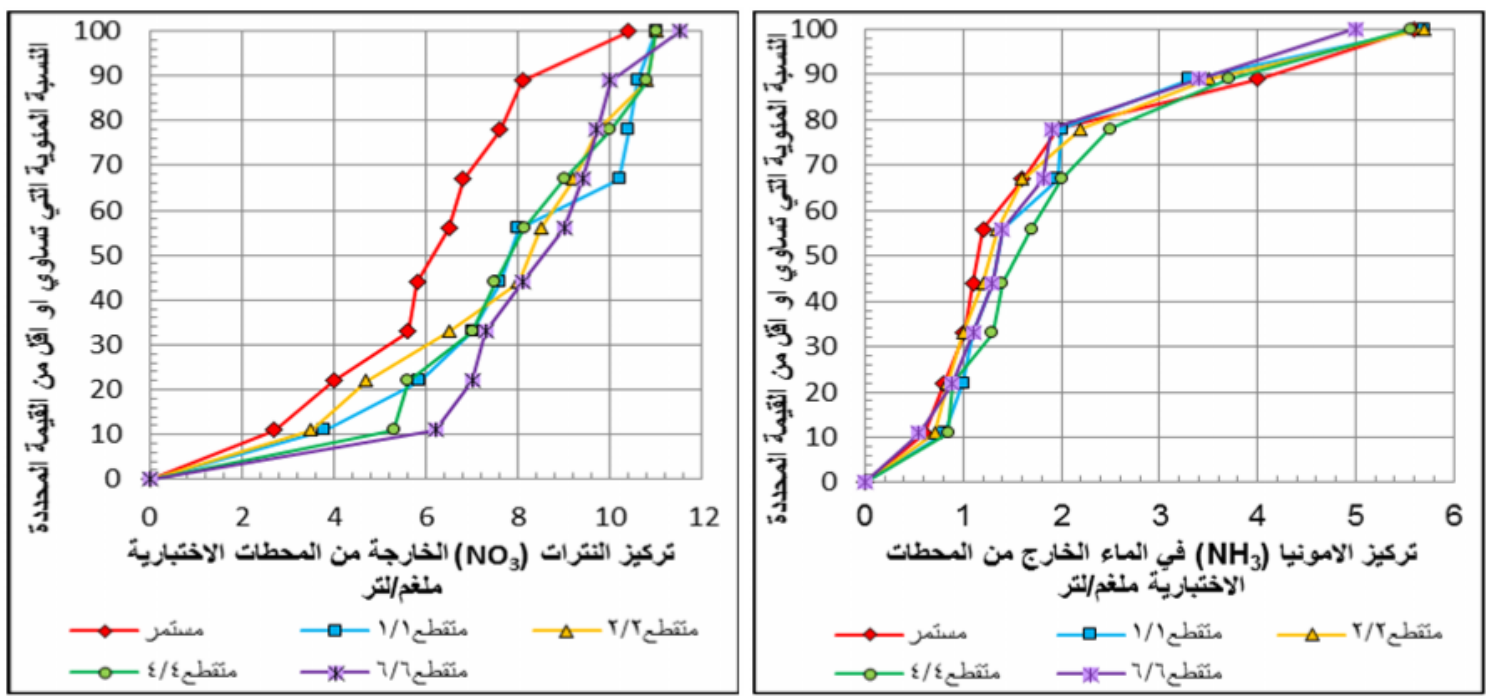

التكل (ד): التوزيع التكراري التراكي لمعدل تراكيز التكل (6): التوزيع التكراري التراكي لمعل تراكيز

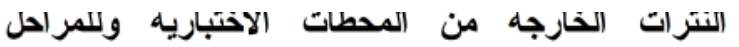

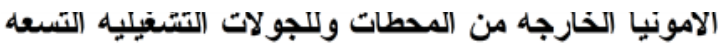
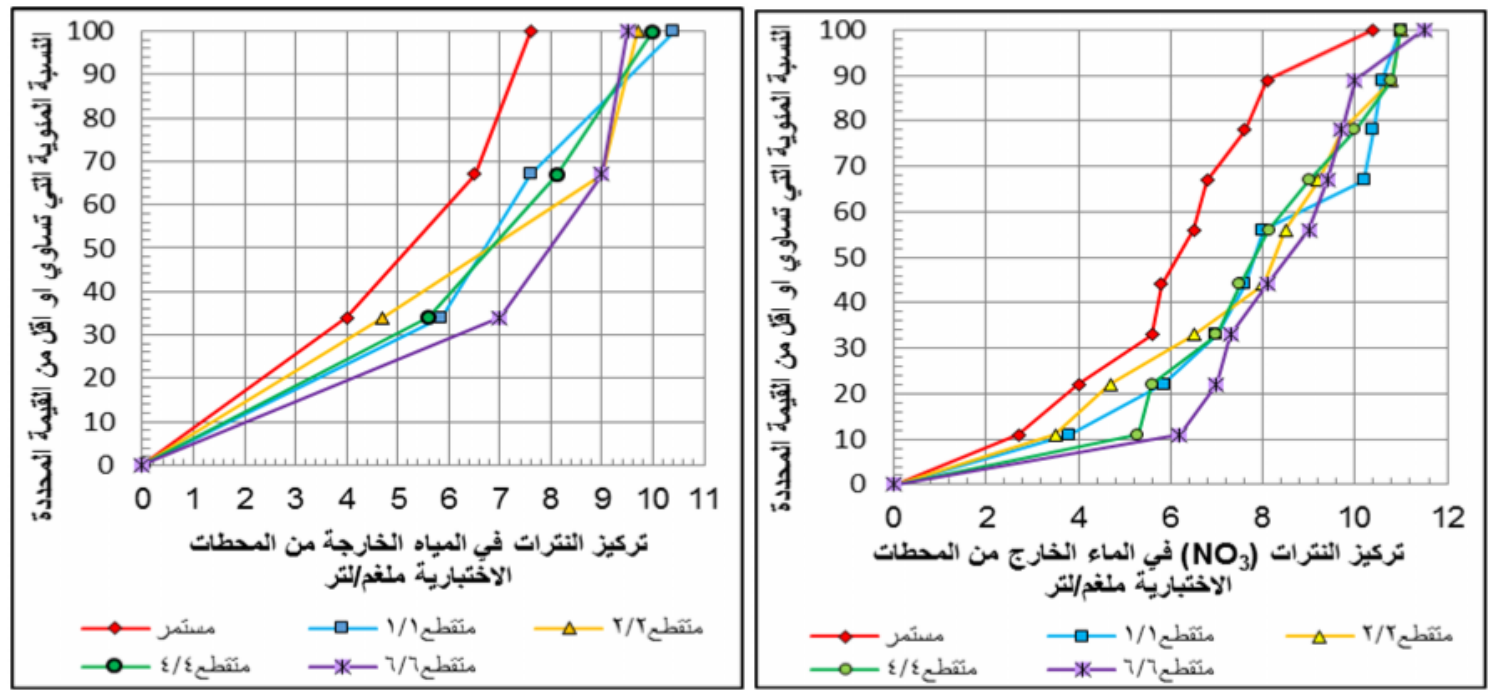

التكل (9): التوزيع التكراري التراكمي لمعدل تراكيز التُكل (8): التوزيع التكراري التراكي لمعدل تراكيز النترات الخارجه من المحطات في الجولات دات التهويه النترات الخارجه من المحطات في الجولات دات التهويه 

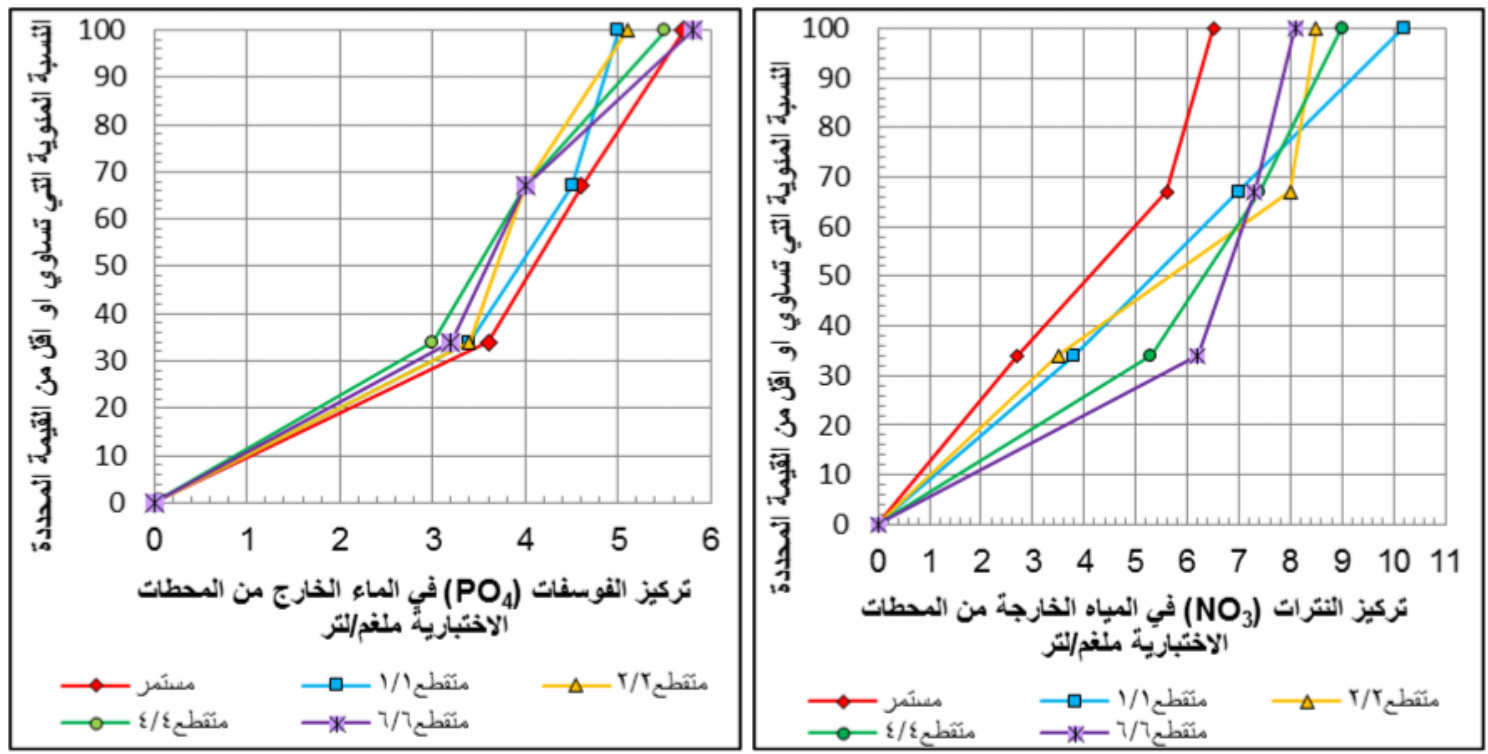

التّكل (11): التوزيع التكراري التراكمي لمعدل تراكيز القوسفات الخارج من المحطات الاختباريه في الجولات يات التهويه المستمره التكل (10): التوزيع النكراري التراكي لمعدل تراكيز النترات الخارجه من المحطات في الجولات دات التهويه المتفطعه بنسبه (3/3)
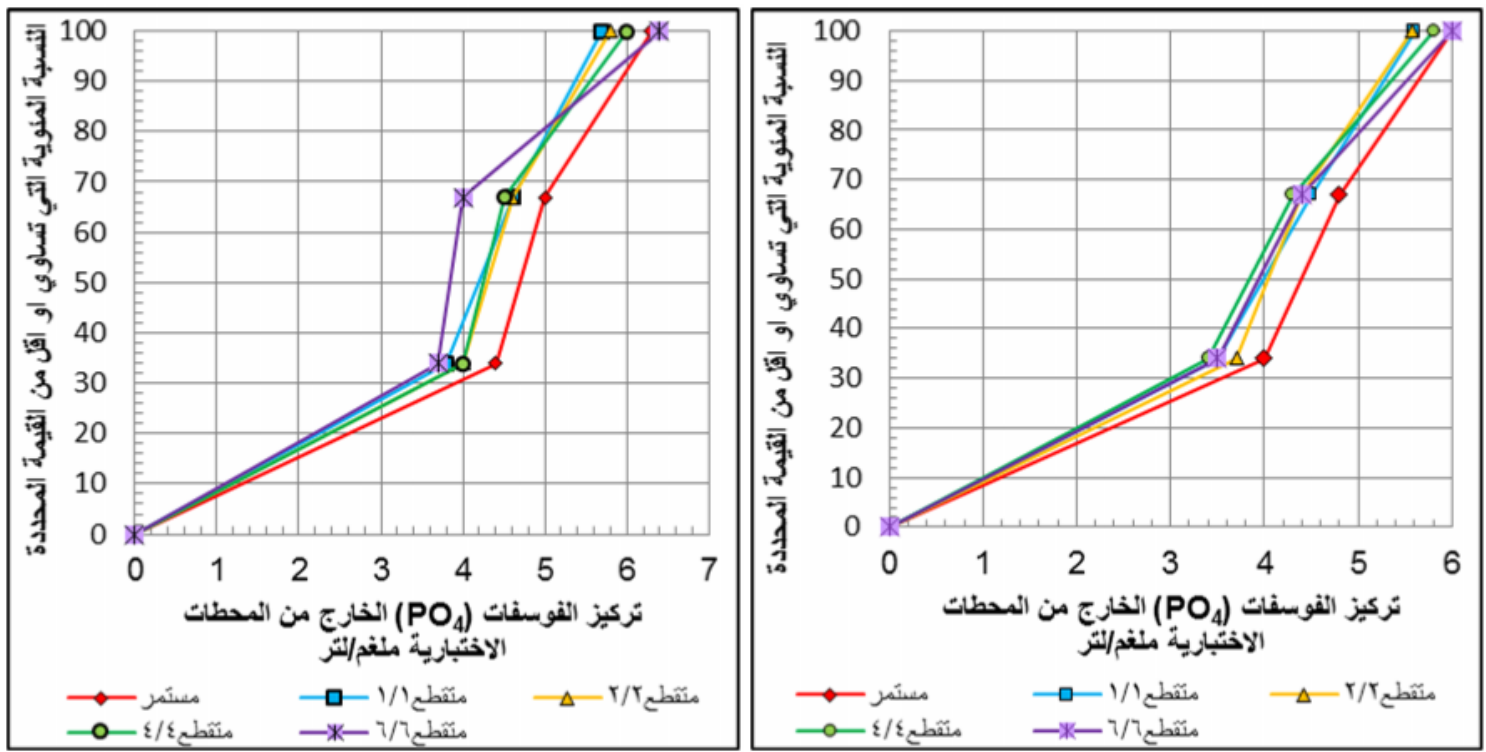

التّكل (13): التوزيع التكراري التراكمي لمعدل تراكيز

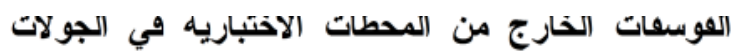
يات التهويه المتفطعه بنسبه (3/3)

التكل (12): التوزيع التكراري التراكمي لمعدل تراكيز

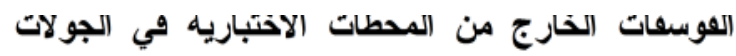
يات التهويه المتفطعه بتسبه (1/1)

الاستتنتاجات

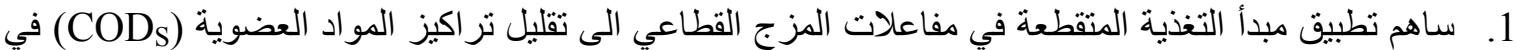

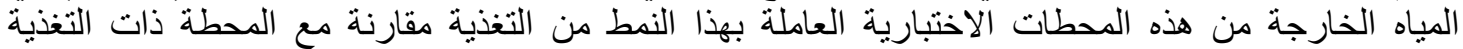
المستمرة وللمر احل التشغيلية الثلاثة. 
2. لوحظ ان تر اكيز النترات في المياه الخارجة من المحطات ذات التغذية المتقطعة كانت اعلى من المياه الخارجة

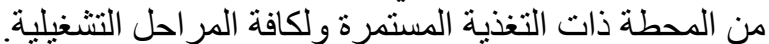

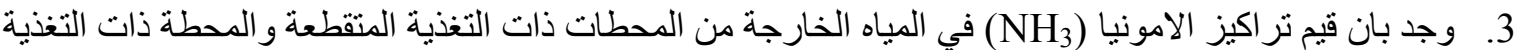

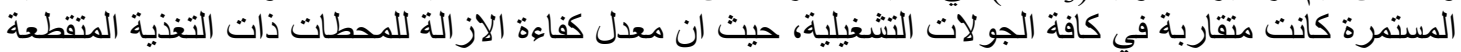

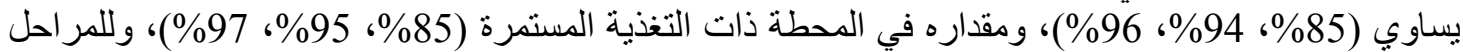
التشغيلية الثلاثة على التو الي.

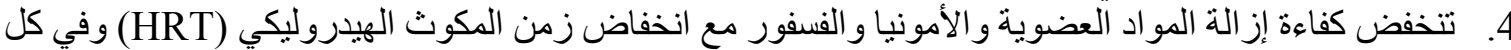
من المفاعلات البيولوجية التي تعمل بنظام التخذية المتقطعة و المفاعل ذات التيات التغذية المستمرة.

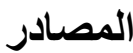

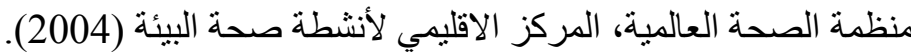

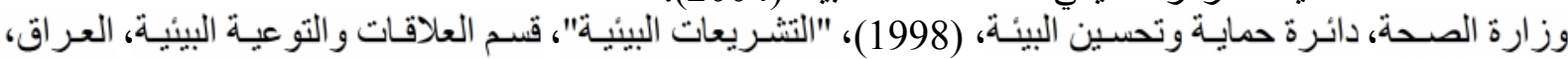
صفحة: 113 -113.

Aivazidis A., Kantartiz S.G. (2004), "Intermittent Feeding Vs Conventional Activated Sludge Treatment For Carbon and Nitrogen Removal ", Protection And Restoration Of The Environment VII Wastewater Treatment and Management I.

Aljarjary A. A.F., (2009), "The Comparison Between Modes of Continuous Aeration and Intermittent Aeration in The Efficiency And Stability of The Continuous Flow Activated Sludge Systems" .

APHA, (1998), "Standard Methods for the Examination of Water and Wastewater", 20th ed., Washington, D.C.

Bracklow U., Drews A., Vocks M., and Kraume M., (2007), "Comparison Of Nutrients Degradation In Small Scale MBR Fed With Synthetic/Domestic Wastewater", Hazardous Materials, Vol. 144 ,No. 3, PP. 620-626.

Chelliapan S., Alkarimiah R., Mahat S. B., Yuzir A., and Din M. F., M., (2011), " Operational Start-Up Performance of an Innovative Anaerobic Stage Reactor (ASR) using Synthetic Wastewater", International Conference on Environment and Industrial Innovation IPCBEE vol.12 (2011) IACSIT Press, Singapore.

Cheng J. and Liu B., (2001), "Nitrification/Denitrification in treatment aeration process for Swine Wastewater treatment", J. of Environmental Engineering, Vol. 127, No. 8.

Chudoba J., Ottova V., Madera V., (1973), "Control Of Activated Sludge Filamentous Bulking -Effect Of The Hydraulic Regime Or Degree Of Mixing In An Aeration Tank", J. of Water Research Vol. 7, Issue 8, PP 1163-1182.

Eckenfelder W.W. (2000) "Industrial water pollution control", 3rd ed., McGraw-Hill, New York.

Ekama G.A., and Wentzel M.G., (1999), "Difficulties and Development in Biological Nutrient Removal Technology and Modeling", Water Science Technology, Vol. 39, Issue 6, PP. 1-13.

EPA, ( 1997), "Wastewater treatment manuals Primary, Secondary and Tertiary treatment".

Fang H.H.P., And Yeong C.L.Y., (1993), "Biological Wastewater Treatment In Reactors With Fibrous Packing", J. Environ. Eng., Vol. 119, Issue 5, PP 46-957.

García-Encina P.A. , García M.C., Mate S. , Adrados F. , Iglesia D. , Fdz-Polanco F. and Villaverde S.,(2002)," Simultaneous Removal Of Organic Matter, Total Nitrogen And 
Phosphorous In A Single Continuous Flow Activated Sludge Reactor Operated With Short Aeration Cycles", e-mail: pedro@iq.uva.es.

Guo J., Xu Y., Chen Y., Fan L., And Fang F., (2012), "Long Term Evolution Of Activated Sludge System Under Low Dissolved Oxygen Conditions", J. Of Environmental Biologe.

Hartley K. J. (1988), "Operating of activated sludge process", third edition, cartridge Haskins and Davey, Austraila.

Hasar H., Kinacib C. , Ünlüa A., To rulc H., and Ipek U., (2002), " Role of Intermittent Aeration in Domestic Wastewater Treatment by Submerged Membrane Activated Sludge System", Desalination, Vol. 142, PP. 287-293.

Kalyuzhngis S., Gladchenko M., Mulder A., And Versprille B., (2007), "Comparison Of Quasistead State Performance Of The DEAMOX Process Under Intermittent And Continuous Feeding And Different Nitrogen Loading Rates"Depatment Of Chemical Enzymology, Chemistry Faculty, Moscow State University, Russian Federation.

Lorestani A.A., Bashiri H., Asadi A., Bonakdgri H., (2012), "Comparison of Different Fluid Dynamics In Activated Sludge System for The Treatment of Astimulated Milk Processing Wastewater: Process Analysis And Optimization", Korean Journal Of Chemical Engineering, Vol.29, Issue 10, PP 1352-1361.

Metcalf and Eddy, Inc. (2003), "Wastewater engineering treatment /disposal /reuse", $4^{\text {th }}$ ed., McGaw-Hill, Inc., New York.

Mortazavi S.B. , Khavanin A. , Moussavi G. and Azhdarpoor A. (2008) ," Removal of Sodium Dodecyl Sulfate in an Intermittent Cycle Extended Aeration System" „Pakistan Journal Biological Sciences 11, Issue 2, PP. 290-293.

Obaja D., Mace S. , Costa J., Sans C., and Mata-Alvarez J., (2002), "Nitrification, Denitrification and Biological Phosphorus Removal in Piggery Wastewater Using a Sequencing Batch Reactor ", Bioresource Technology, Vol. 87, PP 103-111.

Pfenosil J. E., (2003), "Biological Reaction Engineering", Second edition, WILEY-VCH Verlag GmbH \& CO. KGaA, Weinheim.

Ramalho R.S., (1977), "Introduction to wastewater treatment process", Academic press, Inc., Canada.

Russell D.L., (2006), "Practical Wastewater Treatment", Wiley \& Sons, Inc., Hoboken, New Jersey, Published simultaneously in Canada.

Tay J.H., Liu Y., And Liu Q.S., (2001), "Microscopic Observation Aerobic Granulation in Sequential Aerobic Sludge Blanket Reactor", J. Of Applied Microbiology, Vol.19, PP 168175.

Tsai M.-W., Wentzel M.C., and Ekama G.A., (2004), "The Effect of Residual ammonia concentration under aerobic conditions on the growth of M.parvicella in Biological Nutient Removal Plants", Water Research Group, pp. 1078-1086.

Wei Y., Vanhouten R.T., Borger A.R., Eikelboom D.H., And Fan Y., (2003), "Comparison Performance Of Membrance Bioreactor And Continuous Activated Sludge Processes On Sludge Reduction Induced By Oligochaete", Environ. Sc. Technol., Vol. 37, Issue 14, PP 3171-3180.

$$
\text { تم اجراء البحث في كلية ألهندسة = جامعة ألموصل }
$$

